\title{
A Novel Indicator for Quantitative Assessment of Articular Cartilage Degeneration Using Ultrasound
}

\author{
Jing-chen ZHANG ${ }^{1}$, Qiong $\mathrm{HE}^{2}$, Zhi-xiu HAO ${ }^{1, a,{ }^{*}}$, Jian-wen $\mathrm{LUO}^{2}$ \\ and Li-ying $\mathrm{XIAO}^{1, *}$ \\ ${ }^{1,2}$ Department of Mechanical Engineering, Tsinghua University, Beijing, China \\ ahaozx@tsinghua.edu.cn \\ ${ }^{*}$ Corresponding author
}

Keywords: Ultrasound, Cartilage, Backscatter, Quantitative, Degeneration.

\begin{abstract}
Assessment of articular cartilage degeneration induced by degenerative diseases using quantitative ultrasound method is promising but challenging. Currently, in literature, proposed methods have limited diagnostic abilities, and some quantitative ultrasound cartilage evaluation results are inconsistent or hard to compare with each other. Thus it is desirable to find a parameter which is easier to compare and can sensitively reflect changes due to cartilage degeneration. In the present study, a new parameter "averaged magnitude ratio" (AMR) was defined as an indicator to detect articular cartilage degeneration. In vitro experiments were conducted on 12 cartilage-bone samples from porcine knee joints. Six of the samples were digested using $0.25 \%$ trypsin solution. Ultrasound V $(\mathrm{z}, \mathrm{t})$ signal was collected from the samples using a broadband ultrasound transducer with center frequency of $15 \mathrm{MHz}$. Integrated reflection coefficient (IRC), apparent integrated backscattering coefficient (AIB) and AMR were computed from ultrasound signal. Young's modulus of the samples was measured using atomic force microscopy (AFM). With Mann-Whitney U test, statistically significant decrease in IRC and increase in AMR were observed after digestion. With Pearson's correlation analysis, Young's modulus was significantly correlated with AMR, IRC, and AIB. Our results demonstrate that AMR is sensitive to enzymatically induced cartilage changes. AMR is easier to compute, needs no reference material and can achieve equivalent or even better performance than IRC and AIB in detecting cartilage degeneration.
\end{abstract}

\section{Introduction}

Degenerative diseases of articular cartilage like osteoarthritis (OA) are prevalent among elderly people and seriously influence joint function and life quality[1]. OA often leads to fibrillation, softening, ulceration and even loss of cartilage. In order to detect OA-induced degenerative changes in hyaline cartilage, different methods have been proposed, including arthroscopy, MRI, ultrasound, etc. [2, 3]

Ultrasound methods have the potential to assess the state of cartilage degeneration nondestructively. Conventional ultrasonography can reveal structural changes of joint due to osteoarthritis, but it is limited by low resolution and the diagnostic is highly subjective. Quantitative ultrasound methods have also been investigated to detect degenerative changes with higher sensitivity and accuracy. However, despite recent advances, challenges still remain in this field. Acoustic parameters that characterize articular cartilage introduced in previous studies include integrated reflection 
coefficient (IRC) [4], ultrasound roughness index (URI) [5], reflection coefficient (R) [6], broadband ultrasound (US) attenuation [7], apparent integrated backscattering coefficient (AIB) [4], sound speed, cartilage thickness, US aggregate modulus [8], etc. Most of these parameters concern attenuation of reflected acoustic signal from articular surface or matrix relative to acoustic signal reflected from a reference material, or concern cartilage profile. It has been demonstrated that degenerated articular cartilage usually have decreased reflection/backscatter indexes like IRC and R, and increased roughness indexes like URI, due to surface fibrillation and cartilage softening. However, regarding some other acoustic parameters, inconsistent results are found in literature.

In the perspective of acoustic data acquisition in quantitative ultrasound research, most previous studies used intravascular ultrasound device [8] or scanning ultrasound system with an A-mode [4, 5, 8] or B-mode [10] ultrasound transducer. The disadvantage of intravascular method is that it cannot be carried out non-invasively. While it might be possible to examine articular cartilage on patellar surface of femur non-invasively using A or B-mode ultrasound transducer, accurate measurement usually requires normal incidence of ultrasound to the cartilage surface. According to some previously suggested data acquisition method, adjustments are made to ensure perpendicularity at the starting point. However, since the natural articular surface is not flat, when the transducer or the sample moves horizontally to collect data from other positions, perpendicularity may be undermined. In comparison, it may be easier to ensure perpendicularity in the whole data collecting process by moving the transducer vertically. On the other hand, a common disadvantage of methods which involve a reference material is that the reference material is assumed by many studies as a perfect planar reflector, while actually it is not. Different studies may use reference materials with different properties, which brings difficulties for comparison between studies.

The objective of the present study is to find a quantitative parameter which can detect degeneration of articular cartilage and overcome the disadvantages of previously suggested parameters mentioned above. We suggest a novel acoustic parameter averaged magnitude ratio (AMR) as a possible indicator of articular cartilage degeneration. Ultrasound and atomic force microscopy (AFM) measurements were carried out on porcine cartilage in vitro. Young's modulus and acoustic parameters including AMR, IRC and AIB of healthy and trypsin-digested articular cartilage were compared, and the correlation between the parameters was explored.

\section{Materials and Methods}

\section{Specimen Preparation}

Six mature porcine left hind knee joints without visible lesions were obtained from a local market and stored at $-20^{\circ} \mathrm{C}$ until further preparation. For each knee joint, 2 cylindrical cartilage-bone samples $(\Phi=8 \mathrm{~mm}, \mathrm{n}=6 \times 2)$ were prepared from adjacent positions on patellar surface of femur using a hollow drill, and were arranged in the control group and trypsin digestion group respectively.

Samples of the digestion group $(n=6)$ were immersed in $0.25 \%$ trypsin-EDTA solution in a plastic multiwall array. Trypsin digestion of cartilage is often used as a simulation of OA, because trypsin digest proteoglycans, and it also has a slight effect in attacking collagen molecules $[11,12]$. Control samples were immersed in phosphate buffered saline (PBS) in another multiwall array. All samples were kept in an incubator 
(Zhongxingwy, Beijing, China) at $37^{\circ} \mathrm{C}$ for 4 hours. After incubation, samples were embedded in PMMA.

\section{AFM Indentation}

Before indentation measurement, samples were thawed in PBS at room temperature for 1h. A commercial AFM (Dimension Icon, Bruker, US) is used in this study. AFM indentation testing was performed using pyramidal probe tip (SNL 10) with a nominal tip radius of $2 \mathrm{~nm}$ and a spring constant between 0.175 and $0.7 \mathrm{~N} / \mathrm{m}$. The experimental value of spring constant of each cantilever was determined through thermal tune method. Samples were immersed in PBS during indentation. For each sample, 4 regions of $100 \mu \mathrm{m} 2$ near the center of the sample surface was selected, and 25 force-displacement curves were recorded in $5 \times 5$ grid in each of the 4 regions. Thus a total of 100 force curves were recorded for each sample. Ramp size and ramp rate were set to $3 \mu \mathrm{m}$ and $2.06 \mathrm{~Hz}$. Young's modulus was calculated using NanoScope Analysis software (Version 1.50, Bruker, US).

To reduce measurement noise, force-separation curves were filtered by a boxcar filter with average points set as 3. Indentation Analysis was made using Linearized Sneddon model (eq. (1)) [13]. Max and minimum force fit boundary was set as $70 \%$ and $10 \%$.

$$
F=\left(\frac{2 E}{\pi\left(1-v^{2}\right)} \tan (\alpha)\right) \delta^{2} \text {. }
$$

Where $\mathrm{F}$ is force, $\mathrm{E}$ is Young's modulus, $v$ is Poisson's ratio ( $v=0.1$ was used [14]), $\alpha$ is the half-angle of the indenter ( $\alpha=20$ in the present study), and $\delta$ is the indentation depth. Young's modulus was calculated as a quantification of mechanical property of the cartilage.

\section{Ultrasound Measurements}

The experimental setup of the measurement system is shown in Fig 1. A single array single focus ultrasound transducer with nominal center frequency of $15 \mathrm{MHz}$ (V319, Olympus Corporation, Japan) was used. The transducer has a focal length of $25 \mathrm{~mm}$ and a $-6 \mathrm{~dB}$ bandwidth of $11.06-18.91 \mathrm{MHz}$. A pulser-receiver (5073PR, Olympus Corporation, Japan) is used for incident ultrasound pulse generation and echo RF signal acquisition. The RF signal is then digitized at a sampling rate of $2 \mathrm{GHz}$ using a digital oscilloscope (DSO-X 2014A, Agilent Technologies, US) and stored in PC for off-line analysis. The sample was fixed in a container, immersed in PBS solution. The container was fixed on a platform which can move in $\mathrm{x}, \mathrm{y}$ and $\mathrm{z}$ direction under the order of the motion controller (Zolix, Beijing, China).

The region-of-interest for collecting radiofrequency (RF) signals was selected as a square with $100 \mu \mathrm{m}$ width on the center of the sample, within $1 \mathrm{~mm}$ distance from the sampling positions of AFM indentation. On each $\mathrm{Z}$ position, the ultrasound transducer moves in the $\mathrm{X}-\mathrm{Y}$ direction to collect RF signals from nine scan lines $(3 \times 3$ grid $)$, the distance between two scan lines is $50 \mu \mathrm{m}$. Then the transducer moves in the $\mathrm{Z}$ direction towards the sample surface by $23 \mu \mathrm{m}$ and then collect echo signals from the same nine horizontal positions. Before the first measurement, the transducer is adjusted to ensure perpendicularity between the incident sound beam and sample surface, and to set a distance of around $28.5 \mathrm{~mm}$ between the lens and the sample surface. For each sample, data was collected from a total of 400 vertical positions, denoted as $z_{i}(i=1,2, \ldots, 400)$, corresponding to a distance of $9.177 \mathrm{~mm}$ in $\mathrm{z}$ direction. Thus, nine sets of $\mathrm{V}(\mathrm{z}, \mathrm{t})$ signals $[8,15]$ were collected for each sample. 
RF signals were analyzed with custom-designed Matlab R2015b (The Mathworks Inc., Natick, MA, USA) programs. Hilbert transform was performed to get the envelope

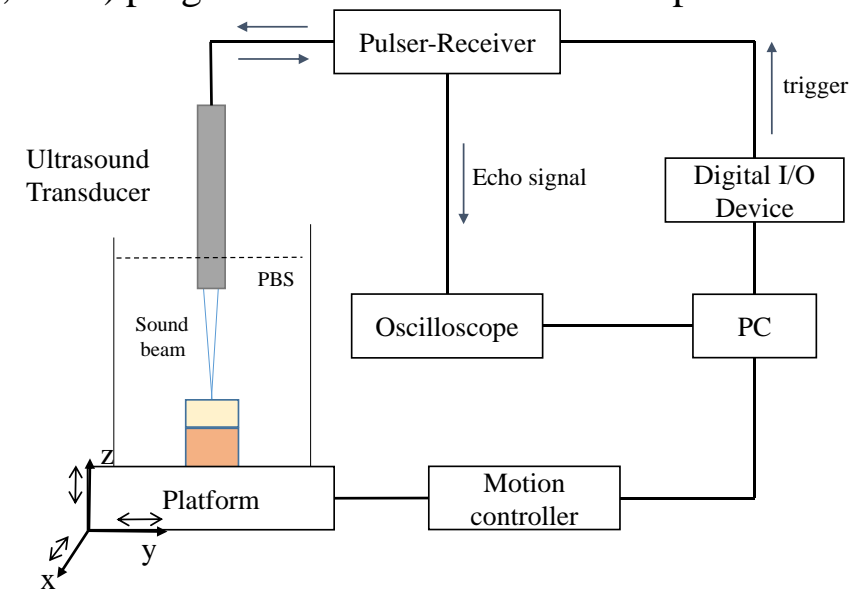

Figure 1. Schematic presentation of the measurement system. During measurement, after the platform has moved to a target position, the digital I/O device produces a pulse signal to trigger the pulser-receiver. Then the transducer emits ultrasound pulse signal and collects the reflected echo signal under the control of the pulser-receiver. The echo data is digitized by the oscilloscope, which is synchronized with the pulser-receiver, and stored in PC. When data storage is finished, the PC and motion controller controls the platform to move to the next target position.

of the reflected signal, and time-of-flight was determined from the location of the maximum values of the envelope. For each set of $\mathrm{V}(\mathrm{z}, \mathrm{t})$, the vertical position $\mathrm{z}_{\mathrm{f}}$ is defined as the transducer's focal point is on the cartilage surface, and $\mathrm{z}_{\mathrm{f}}$ is determined as where the largest PBS-cartilage interface echo envelope magnitude is found. The nine $z_{f}$ positions of a same sample can be slightly different, and the nine scan signals collected in the X-Y plane with the max $z_{f}$ position were used to determine IRC and AIB $[8,15]$ of saline-cartilage interface, according to formulae in Table 1. A polished stainless steel plate was used as reference material.

AMR was determined from $V(z, t)$ signals in a certain depth zone as follows:

$$
A M R=\frac{\int_{\Delta z}\left(\frac{1}{L_{z, l}} \int_{L_{z, l}}|V(z, t)| d t+\frac{1}{L_{z, r}} \int_{L_{z, r}}|V(z, t)| d t\right) d z}{\int_{\Delta z} \frac{1}{L_{z, m}} \int_{L_{z, m}}|V(z, t)| d t d z} \cdot \square \square \square \square \square \square
$$

For digitized signal, we can use the discrete form:

$$
A M R=\frac{\sum_{i=m_{z}}^{m_{z}+M_{z}}\left(\frac{1}{M_{l}} \sum_{j=m_{i}^{l}}^{m_{i}^{l}+M_{l}}\left|V_{i j}\right|+\frac{1}{M_{r}} \sum_{j=m_{i}^{r}}^{m_{i}^{r}+M_{\mathrm{r}}}\left|V_{i j}\right|\right)}{\sum_{i=m_{z}}^{m_{z}+M_{z}}\left(\frac{1}{M_{m}} \sum_{j=m_{i}^{m}}^{m_{i}^{m}+M_{m}}\left|V_{i j}\right|\right)} .
$$

where $\mathrm{L}_{z, 1}, \mathrm{~L}_{z, \mathrm{r}}, \mathrm{L}_{z, \mathrm{~m}}$ are specified sections of signal colleceted from a certain scan line. $\mathrm{m}_{\mathrm{i}}{ }^{1}, \mathrm{~m}_{\mathrm{i}}{ }^{\mathrm{r}}$ and $\mathrm{m}_{\mathrm{i}}{ }^{\mathrm{m}}$ are the starting index of section $\mathrm{L}_{z, 1}, \mathrm{~L}_{\mathrm{z}, \mathrm{r}}, \mathrm{L}_{\mathrm{z}, \mathrm{m}}$, respectively. $\mathrm{M}_{1}, \mathrm{M}_{\mathrm{r}, \mathrm{and}}$ a $M_{m}$ are the number of data points in section $L_{z, l}, L_{z, r}, L_{z, m}$, respectively. $\Delta z$ is the vertical distance of the chosen depth zone, $\mathrm{m}_{\mathrm{z}}$ is the starting index of the depth zone, $\mathrm{M}_{\mathrm{z}}$ is the number of vertical positions in the chosen depth zone, $\mathrm{V}$ denotes the value of acquired signal at the corresponding data point. 
Fig. 2 illustrates the symbols of parameters needed for AMR determination. Average echo signal magnitude between the saline-cartilage interface and the cartilage-bone interface was measured. The vertical position which maximizes this average magnitude is determined as the center of the depth zone $\mathrm{z}_{\mathrm{c}}$. The signal sections $\mathrm{L}_{\mathrm{z}, \mathrm{el}}, \mathrm{L}_{\mathrm{z}, \mathrm{er}}, \mathrm{L}_{\mathrm{z}, \mathrm{l}}, \mathrm{L}_{\mathrm{z}, \mathrm{r}}$ and $\mathrm{L}_{\mathrm{z}, \mathrm{m}}$ used for AMR determination can be adjusted by setting the signal section length proportion $1_{1}, 1_{2}, 1_{3}$ and $1_{4}$. The signal sections are adjusted with respect to the thickness of cartilage $\mathrm{d}$ :

$$
\begin{aligned}
& L_{z, e l}=l_{1} \cdot d, \square \quad \square L_{z, e r}=l \cdot a \\
& L_{z, l}=l_{2} \cdot d \quad, \quad L_{z, r}=l_{3} \cdot d \\
& L_{z, m}=\left(1-l_{1}-l_{2}-l_{3}-l_{4}\right) \cdot d
\end{aligned}
$$

In the present study, results for AMR calculated from different depth zone and signal section proportion are shown. The inter-site variability of AMR was estimated from the nine $\mathrm{V}(\mathrm{z}, \mathrm{t})$ measurements performed on each sample using standard coefficient of variation $(\mathrm{SCV})[16,17]$.
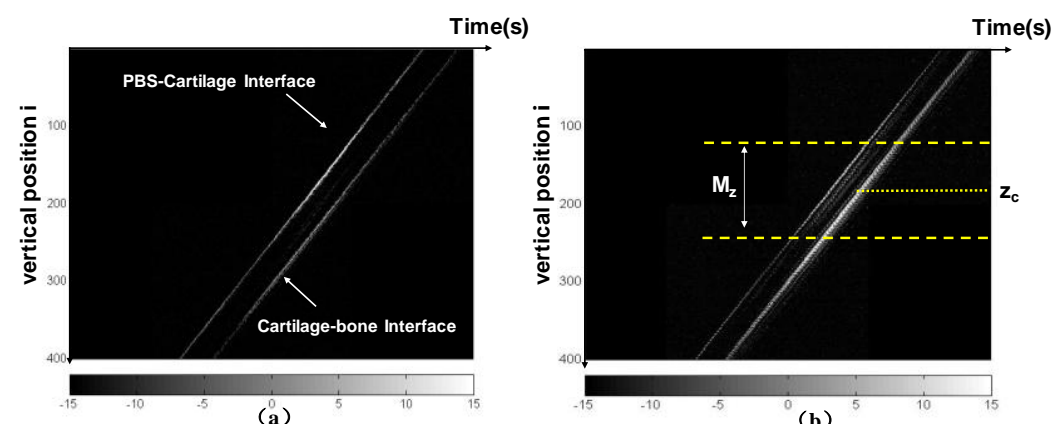

(b)

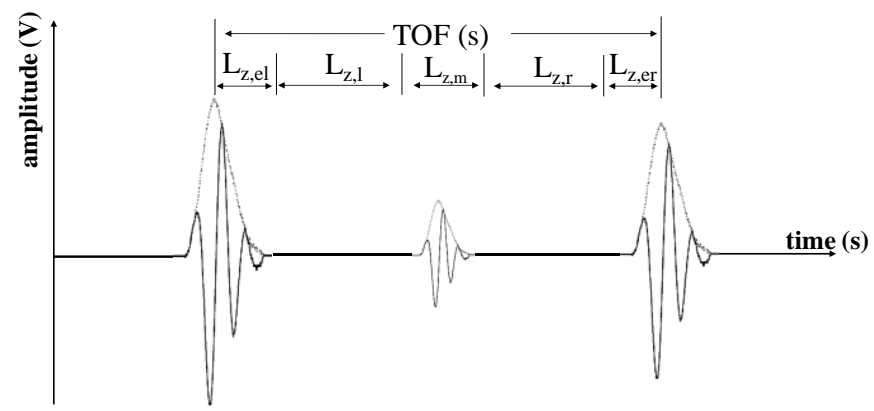

(c)

Figure 2. (a) $\mathrm{V}(\mathrm{z}, \mathrm{t})$ signal from a control sample.

Image intensity represents amplitude of the echo signal. (b) $\mathrm{V}(\mathrm{z}, \mathrm{t})$ signal from a trypsin-digested sample. Depth zone is marked with dashed line, $z_{c}$ is marked with dotted line. (c) diagram of reflected RF signal from a scan line in the chosen depth zone. Signal envelope is shown with dotted line. 
Table 1. The mathematical definitions of parameters evaluated in the present study

\begin{tabular}{|c|c|c|}
\hline IRC & AIB & SCV \\
\hline$I R C=\frac{1}{\Delta f} \int_{\Delta f} 10 \log _{10}\left\langle\mid \frac{S_{C}(z, f)}{S_{R}(z, f)}\right\rangle^{2} d f$ & $A I B=\frac{1}{\Delta f} \int_{\Delta f} 10 \log _{10}\left\langle\left|\frac{S_{C}\left(z_{v}, f\right)}{S_{R}\left(z_{v}, f\right)}\right|\right\rangle^{2} d f$ & $S C V=\frac{C V \cdot \bar{x}_{1 s t}}{4 \cdot S D_{1 s t}}$ \\
\hline
\end{tabular}

SC and SR are the amplitude spectra of the signal reflected from the PBS-cartilage interface and the PBS-stainless steel interface, respectively. $\mathrm{z}=$ distance between the transducer and the cartilage surface; $\mathrm{zV}=$ distance of the evaluated volume from the transducer. $\mathrm{CV}$ is the coefficient of variation, $\bar{x}_{\mathrm{sts}}$ and SD1st are the mean and standard deviation of the first measurement of the samples, respectively.

\section{Statistical Analysis}

Statistical significance of differences in ultrasound parameters and Young's modulus measured by AFM was determined with Mann-Whitney U test. Correlations between acoustic parameters and Young's modulus were evaluated using Pearson's analysis. The limit of significance was set as $\mathrm{p}<0.05$. Statistical analyses were conducted using Matlab R2015b (The Mathworks Inc., Natick, MA, USA).

\section{Results and Discussion}

Mean values $( \pm \mathrm{SD})$ and SCV of ultrasound parameters measured in the present study, as well as their correlation coefficient with Young's Modulus, are presented in Table 2.

Table 2. Mean Values $( \pm \mathrm{SD}), \mathrm{SCV}$ and Correlation Coefficient of Measured Ultrasound Parameters

\begin{tabular}{|c|c|c|c|c|c|c|c|c|c|}
\hline Parameters & $\mathrm{Mz}$ & 11 & 12 & 13 & 14 & Control & $\begin{array}{l}\text { Trypsin- } \\
\text { digested }\end{array}$ & $\begin{array}{c}\text { Correlation } \\
\text { Coefficient with }\end{array}$ & $\mathrm{SCV}$ \\
\hline \multirow{9}{*}{ AMR } & \multirow{3}{*}{41} & 0.1 & 0.5 & 0.1 & 0.1 & $1.91 \pm 0.42$ & $2.78 \pm 0.93 *$ & $-0.590 \%$ & $10.7 \%$ \\
\hline & & 0.15 & 0.5 & 0.1 & 0.1 & $1.90 \pm 0.32$ & $2.70 \pm 0.90^{*}$ & $-0.589 \ddagger$ & $13.3 \%$ \\
\hline & & 0.15 & 0.5 & 0.1 & 0.1 & $1.81 \pm 0.28$ & $2.73 \pm 0.89^{*}$ & -0.592 + & $9.1 \%$ \\
\hline & \multirow{3}{*}{61} & 0.1 & 0.5 & 0.1 & 0.1 & $1.93 \pm 0.40$ & $2.75 \pm 0.91^{*}$ & $-0.592 \ddagger$ & $9.8 \%$ \\
\hline & & 0.15 & 0.5 & 0.1 & 0.1 & $1.92 \pm 0.31$ & $2.67 \pm 0.89^{*}$ & $-0.588 *$ & $11.7 \%$ \\
\hline & & 0.15 & 0.5 & 0.1 & 0.1 & $1.83 \pm 0.27$ & $2.70 \pm 0.86^{*}$ & $-0.592 \ddagger$ & $8.4 \%$ \\
\hline & \multirow{3}{*}{81} & 0.1 & 0.5 & 0.1 & 0.1 & $1.94 \pm 0.39$ & $2.73 \pm 0.88^{*}$ & $-0.589+$ & $8.9 \%$ \\
\hline & & 0.15 & 0.5 & 0.1 & 0.1 & $1.93 \pm 0.30$ & $2.64 \pm 0.87 *$ & $-0.580 \ddagger$ & $10.2 \%$ \\
\hline & & 0.15 & 0.5 & 0.1 & 0.1 & $1.84 \pm 0.26$ & $2.67 \pm 0.84 *$ & $-0.590 \$$ & $7.9 \%$ \\
\hline & \multicolumn{9}{|c|}{ Time window length } \\
\hline $\mathrm{AIB}(\mathrm{dB})$ & \multicolumn{3}{|c|}{0.45 us } & & & $-58.83 \pm 2.6$ & $-51.98 \pm 7.7$ & $-0.605+$ & \\
\hline $\operatorname{IRC}(\mathrm{dB})$ & \multicolumn{3}{|c|}{0.3 us } & & & $-19.06 \pm 0.5$ & $-26.41 \pm 2.22^{*}$ & $0.637 \dagger$ & \\
\hline
\end{tabular}

${ }^{*} \mathrm{p}<0.05$ for Mann-Whitney U test;

${ }^{\ddagger} \mathrm{p}<0.05$ for Pearson's correlation test

In the present study, we proposed a new parameter AMR as an indicator of articular cartilage degeneration. According to our experiments, with the different depth zones and signal sections we selected, significant increase in the value of AMR was found after trypsin digestion, and significant correlations exist between AMR and Young's modulus. Since elastic property is essential to articular cartilage function, it is desired that changes in Young's modulus can be detected sensitively. It can be seen that AMR is sensitive to cartilage degeneration, and performs well in reflecting elastic property of cartilage. Significant decrease in IRC after trypsin-digestion, and significant correlation between IRC and Young's modulus can was also observed. However, the difference of AIB between the two sample groups did not reach the significant level. AMR has acceptable spatial variation, with SCV between $7.9 \%-13.3 \%$. 
As is shown in Figure 2, for healthy samples, cartilage matrix near the cartilage surface and near the cartilage-bone interface was almost anechoic compared with the two interfaces and the middle part of the cartilage matrix. The middle part is relatively hyperechoic, probably because this part is near the transitional zone, where collagen content, fibril orientation as well as chondrocyte size and shape are subject to change [18]. However, for degraded samples, upper and deep parts of the cartilage matrix are also likely to be hyperechoic, thus degraded samples show higher AMR compared to controls. The reason might be: i) roughness of cartilage surface increases as the cartilage degrades $[5,19]$, thus less energy is reflected from the cartilage surface, more energy was able to penetrate into the cartilage matrix and be reflected or backscattered from different depths. ii) Collagen fibrils in degenerated cartilage matrix are more randomly aligned, and the distribution of chondrocytes may be changed due to collagen network disruption, which is likely to induce more dispersed backscatter of acoustic waves.

For calculation of many acoustic parameters proposed in previous studies like IRC, AIB and $\mathrm{R}$, a reference signal reflected from a reference specimen under the same experimental condition needs to be obtained. However, the reference reflector may not be identical in different studies. For example, in some previous studies the reference signal may be collected from PBS-air interface [5], sodium chloride-silicon rubber interface [8], PBS-PMMA interface [20] or physiologic saline-steel interface [17]. Also, assumptions are usually made that the acoustic characteristics of the reference material are known and fixed, but more or less, the real acoustic characteristics of reference material deviate from what has been assumed. Even with similar reference material, in different studies, these deviations may be quite different. This brings difficulties for summarizing different studies. For example, in literature, for healthy bovine patellae cartilage, the reported mean value of IRC measured under normal incidence by $40 \mathrm{MHz}$ transducer varies from around $-17.7 \mathrm{~dB}$ [17] to around $-23 \mathrm{~dB}$ [21]. The calculation of AMR does not need a reference signal, which is advantageous for comparison between studies and eliminates error induced by reference material.

On the other hand, due to the natural curvature of articular cartilage surface, as the scan line moves horizontally relative to the sample surface for the measurement of IRC and AIB, the perpendicularity between sound beam and the sample surface is likely to be undermined. For the measurement of AMR, it is easier to ensure the perpendicularity and may introduce less error since the transducer moves vertically. Furthermore, in most previous studies, the distance between the transducer and sample surface was largely fixed, thus observation of cartilage matrix with larger defocus distance is not optimal. In the acquisition of $\mathrm{V}(\mathrm{z}, \mathrm{t})$ data, as the focus of sound beam moves vertically across the cartilage layer, more information from the entire cartilage layer can be obtained, which may provide better insight of the changes of samples. This might explain AMR's stronger ability to discern the two sample groups, and better correlation with Young's modulus than AIB.

It is shown that with proper selection of the signal length section proportion $\left(1_{1}, 1_{2}, 1_{3}\right.$, $1_{4}$ ) and depth zone $\mathrm{M}_{\mathrm{z}}$, AMR can achieve a good performance in detecting cartilage degeneration. The measurement of AMR may contribute to noninvasive ultrasound examinations from outside of the joint for diagnosis of diseases like OA, which involve cartilage degeneration. As this is only a preliminary report, we measured only 12 samples. In our further studies, the diagnostic potential of this novel parameter will be investigated with larger sample size and with different states of degeneration. 


\section{Conclusion}

Results of the present study suggest that the AMR is able to sensitively detect cartilage degeneration induced enzymatically with trypsin. It showed some advantages compared with AIB, which is not sensitive enough to distinguish the two groups. Furthermore, AMR has some advantages regarding perpendicularity compared with AIB and IRC, and no reference material is needed for calculation of AMR. The method suggested in the present study may provide more insight into cartilage degeneration detection.

\section{Acknowledgement}

This research was supported by the National Science Foundation (51275267).

\section{References}

[1] S. Knecht, B. Vanwanseele and E. Stüssi, Clin Biomech, vol. 21, (2006) 999-1012.

[2] P. Kiviranta, J. Toyras, M. T. Nieminen, M. S. Laasanen, S. Saarakkala, H. J. Nieminen, M. J. Nissi and J. S. Jurvelin, Eur Cell Mater, vol. 13, (2007) 46-55; discussion 55 .

[3] A. Iagnocco, Best Practice \& Research Clinical Rheumatology, vol. 24, (2010) 27-38.

[4] E. Cherin, A. Saied, P. Laugier, P. Netter and G. Berger, Ultrasound Med Biol, vol. 24, (1998) 341-54.

[5] S. Saarakkala, J. Töyräs, J. Hirvonen, M. S. Laasanen, R. Lappalainen and J. S. Jurvelin, Ultrasound in Medicine \& Biology, vol. 30, (2004) 783-792.

[6] T. Virén, S. Saarakkala, J. S. Jurvelin, H. J. Pulkkinen, V. Tiitu, P. Valonen, I. Kiviranta, M. J. Lammi and J. Töyräs, Ultrasound in Medicine \& Biology, vol. 36, (2010) 833-839.

[7] H. J. Nieminen, S. Saarakkala, M. S. Laasanen, J. Hirvonen, J. S. Jurvelin and J. Töyräs, Ultrasound in Medicine \& Biology, vol. 30, (2004) 493-500.

[8] A. Sun, X. Bai and B. Ju, Rev Sci Instrum, vol. 86, (2015) 034301.

[9] J. Liukkonen, P. Lehenkari, J. Hirvasniemi, A. Joukainen, T. Virén, S. Saarakkala, M. T. Nieminen, J. S. Jurvelin and J. Töyräs, Ultrasound in Medicine \& Biology, vol. 40, (2014) 2039-2047.

[10] S. Ohashi, I. Ohnishi, T. Matsumoto, M. Bessho, J. Matsuyama, K. Tobita, M. Kaneko and K. Nakamura, Ultrasound in Medicine \& Biology, vol. 38, (2012) 402-411.

[11] C. P. Brown, S. W. Hughes, R. W. Crawford and A. Oloyede, Phys Med Biol, vol. 53, (2008) 4123-35.

[12] S. Saarakkala, S. Wang, Y. Huang, J. S. Jurvelin and Y. Zheng, Ultrasound in Medicine \& Biology, vol. 37, (2011) 112-121.

[13]D. C. Lin, E. K. Dimitriadis and F. Horkay, J Biomech Eng, vol. 129, (2007) 430-40.

[14] L. Han, E. H. Frank, J. J. Greene, H. Lee, H. K. Hung, A. J. Grodzinsky and C. Ortiz, Biophys J, vol. 100, (2011) 1846-1854. 
[15] J. Chen, X. Bai, K. Yang and B. Ju, Rev Sci Instrum, vol. 83, (2012) 014901.

[16]C. Fournier, S. Lori Bridal, A. Coron and P. Laugier, IEEE Transactions on Ultrasonics, Ferroelectrics and Frequency Control, vol. 50, (2003) 408-418.

[17] S. Wang, Y. Huang, S. Saarakkala and Y. Zheng, Ultrasound in Medicine \& Biology, vol. 36, (2010) 512-527.

[18] N. Männicke, M. Schöne, M. Gottwald, F. Göbel, M. L. Oelze and K. Raum, Ultrasound in Medicine \& Biology, vol. 40, (2014) 244-257.

[19]E. Kaleva, S. Saarakkala, J. Töyräs, H. J. Nieminen and J. S. Jurvelin, Ultrasound in Medicine \& Biology, vol. 34, (2008) 155-159.

[20] M. Schöne, N. Männicke, M. Gottwald, F. Göbel and K. Raum, Ultrasound in Medicine \& Biology, vol. 39, (2013) 834-844.

[21]T. Virén, M. Timonen, H. Tyrväinen, V. Tiitu, J. S. Jurvelin and J. Töyräs, Osteoarthr Cartilage, vol. 20, (2012) 719-726. 